\title{
Variabilidade espacial de atributos químicos e de produtividade na cultura do café
}

\author{
Spatial variability of chemical attributes and productivity in the coffee cultivation
}

\author{
Fábio Moreira da Silva $^{\mathrm{I}^{*}}$ Zigomar Menezes de Souza $^{\mathrm{I}}$ Carlos Augusto Pereira de Figueiredo \\ José Marques Júnior ${ }^{I I}$ Rodrigo Villela Machado ${ }^{\mathrm{I}}$
}

\section{RESUMO}

O conhecimento de determinados atributos do solo associado à resposta de produção do cafeeiro pode possibilitar a aplicação racional, localizada e individualizada dos insumos, com resultados econômicos ambientais garantidos. Portanto, o objetivo deste trabalho foi caracterizar a variabilidade espacial de atributos químicos do solo e da produtividade da cultura do cafeeiro em um Latossolo Vermelho-Amarelo distrófico. Amostras de solo foram coletadas na profundidade de 0-0,2m, nos pontos de cruzamento de uma malha com intervalos regulares de $25 \mathrm{~m}$, perfazendo um total de 68 pontos em uma área de 4,2ha. Os frutos de quatro plantas em torno dos pontos de cruzamento da malha foram coletados para o cálculo da produção média por planta. Todas as variáveis estudadas apresentaram estrutura de dependência espacial, o que permitiu o seu mapeamento com a utilização de técnicas geoestatísticas. Os atributos químicos apresentaram valores baixos de alcance, com exceção das variáveis fósforo e potássio, evidenciando a baixa continuidade espacial do solo sob cafeeiro. A grande amplitude de variação dos atributos químicos justifica a aplicação diferenciada e localizada de fertilizantes na lavoura cafeeira.

Palavras-chave: agricultura de precisão, geoestatística, cultura do cafeeiro.

\section{ABSTRACT}

The knowledge of determined attributes of the soil associated to the reply of productivity of the coffee can make the application rational, located and individualized of the farm input, with guaranteed ambient economic results possible. Therefore, this work was aimed at evaluating the spatial variability of soil chemical attributes and the productivity of coffee cropping in an Oxisol. Soil samples were collected in a depth of $0-0.2 \mathrm{~m}$, at the crossing points of a regular grid with 25 m-intervals, comprising a total of 68 points in an area of 6.2ha. Fruits of four coffee plants around the soil sampling points were collected and the average production per plant was calculated. The variables presented a spatial dependence structure, allowing their mapping by geostatistics techniques. Chemical attributes presented low range values, with exception of the phosphorus and potassium variables, evidencing the low soil continuity spatial under the coffee crop. The wide variation range of chemical attributes shows the necessity of point fertilizer application for coffee cropping.

Key words: site-specific management, geostatistics, coffee cropping.

\section{INTRODUÇÃO}

O Estado de Minas Gerais responde por mais de $50 \%$ da produção de café do Brasil, tendo produzido, na safra de 2004/2005, 39,3 milhões de sacas, havendo ainda uma previsão de 33,3 milhões de sacas para a safra de 2005/2006 (CONAB, 2006). Além de ser o maior Estado produtor, Minas Gerais conta atualmente com o parque cafeeiro mais tecnificado, sendo o que mais emprega a colheita mecanizada. Portanto, uma alternativa viável para este setor é o emprego da agricultura de precisão.

Áreas consideradas pedologicamente similares podem apresentar variabilidade distinta em atributos quando submetidas às diferentes práticas de manejo. O manejo pode alterar os atributos químicos, físicos, mineralógicos e biológicos, com impacto principalmente nas camadas superficiais do solo (MARQUES JÚNIOR et al., 2000). Portanto, conhecer

\footnotetext{
IDepartamento de Engenharia, Universidade Federal de Lavras (UFLA), Lavras, MG, Brasil. E-mail:fmsilva@ufla.br.*Autor para correspondência.

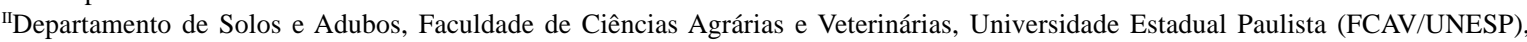
Jaboticabal, SP, Brasil.
} 
a variabilidade espacial dos atributos do solo que controlam a produtividade das culturas é um fator indispensável. Fatores como declividade e posição topográfica influenciam na produtividade das culturas de maneira indireta, por influenciarem nos atributos químicos e físicos do solo (SOUZA et al., 2004).

As aplicações convencionais de fertilizantes atualmente realizadas pelos agricultores implicam aplicações excessivas em determinadas áreas do campo e insuficientes em outras. O conhecimento detalhado da variabilidade espacial dos atributos da fertilidade pode otimizar as aplicações localizadas de corretivos e fertilizantes, melhorando dessa maneira o controle do sistema de produção das culturas, reduzindo os custos gerados pela alta aplicação de insumos e a degradação ambiental provocada pelo excesso destes nutrientes (ROCHA\& LAMPARELLI, 1998).

As pesquisas com agricultura de precisão com a cultura do café têm demonstrado variabilidade acentuada de atributos do solo e da produção. MARQUES JÚNIOR et al. (2000), investigando a variabilidade espacial de atributos químicos e granulométricos do solo de uma lavoura de café na região de Patrocínio-MG, observaram dependência espacial para todos os atributos estudados. Embora não se tenha caracterizado o processo de definição da variabilidade, observaram dependência espacial da produtividade. BALASTREIRE et al. (2001) realizaram um mapeamento da produtividade da cultura do café no município de Pinhal-SP e verificaram variabilidade da produtividade do café, a partir de um mínimo de $1,4 \mathrm{Mg} \mathrm{ha}^{-1}$ a um máximo de $18,4 \mathrm{Mg} \mathrm{ha}^{-1}$, o que corresponde à variação de $12,9 \mathrm{Mg} \mathrm{ha}^{-1}$. MOLIN et al. (2002) realizaram um trabalho de mapeamento de produtividade de café e sua correlação com os componentes de fertilidade do solo em duas áreas pilotos nos municípios de Gália-SP e Pompéia-SP e observaram que os componentes de correlação entre a produtividade e fertilidade do solo resultaram em baixos valores. As duas áreas apresentaram grande variação na produtividade (1,3 a 4,5Mg ha-1).

A aplicação da agricultura de precisão na cultura do cafeeiro ainda não é uma realidade na agricultura brasileira, uma vez que existem poucos trabalhos sendo desenvolvidos nesta área. Portanto, o objetivo deste trabalho foi caracterizar a variabilidade espacial de atributos químicos e a produtividade da cultura do cafeeiro em um Latossolo Vermelho-Amarelo distrófico.

\section{MATERIAL E MÉTODOS}

O experimento foi conduzido na fazenda Cafua, localizada no município de Ijací, sul de Minas Gerais, em uma área de 4,2ha de lavoura da variedade Mundo Novo, com doze anos de idade, plantada no espaçamento de $4 \mathrm{~m}$ entre linhas e de $1 \mathrm{~m}$ entre plantas, totalizando 2.500 plantas ha-1. As coordenadas geográficas são de $21^{\circ} 10^{\prime} 11^{\prime \prime} \mathrm{S}$ e $44^{\circ} 58^{\prime} 37^{\prime \prime} \mathrm{W}$, com altitude média de $934 \mathrm{~m}$ e uma declividade de $0,84 \%$ no sentido longitudinal e de $12 \%$ no sentido transversal. O clima da região foi classificado pelo método de Köppen como subtropical com inverno seco (Cwb).

$\mathrm{Na}$ safra de 2004, foram coletados manualmente sobre panos os dados da produtividade de quatro plantas em torno dos pontos amostrais durante o mês de julho, sendo considerada a produtividade média por planta. Seguindo a faixa de adubação, as amostras de solo foram coletadas com trado na profundidade de $0-0,2 \mathrm{~m}$, em cada um dos pontos georreferenciados. O solo da área foi classificado como Latossolo Vermelho-Amarelo distrófico típico.

Os solos foram amostrados nos pontos de cruzamento de uma malha com intervalos regulares de $25 \mathrm{~m}$, perfazendo um total de 68 pontos em uma área de 4,2ha, na profundidade de $0-0,2 \mathrm{~m}$. Na caracterização química do solo, foram determinados a acidez ativa $(\mathrm{pH}$ em água), determinada potenciometricamente, os teores de cálcio (Ca) e magnésio (Mg) trocáveis, utilizandose o extrator $\mathrm{KCl} 1 \mathrm{~N}$, e os teores de fósforo (P) e potássio (K) disponíveis, extraídos pelo extrator Mehlich-1 (EMBRAPA, 1997). Com base nos resultados das análises químicas, forem calculadas a soma de bases (SB) e a capacidade de troca catiônica (CTC efetiva).

Os atributos químicos do solo e a produção da cultura do café foram analisados por meio da análise estatística descritiva e de técnicas geoestatísticas. A hipótese de normalidade dos dados foi testada pelo teste de Kolmogorov-Smirnov, por meio do programa computacional SAS (SCHLOTZHAVER \& LITTELL, 1997). A dependência espacial foi analisada por meio de ajustes de semivariogramas (VIEIRA et al., 1983), com base na pressuposição de estacionariedade da hipótese intrínseca, a qual é estimada por:

$\hat{\gamma}(h)=\frac{1}{2 N(h)} \sum_{i=1}^{N(h)}\left[Z\left(x_{i}\right)-Z\left(x_{i}+h\right)\right]^{2}$, em que $N$ (h) é o número de pares experimentais de observações $\mathrm{Z}\left(\mathrm{x}_{\mathrm{i}}\right)$ e $\mathrm{Z}\left(\mathrm{x}_{\mathrm{i}}+\mathrm{h}\right)$ separados por uma distância h. O semivariograma é representado pelo gráfico $\hat{\gamma}(h)$ versus h. Do ajuste de um modelo 
matemático aos valores calculados de $\hat{\gamma}(h)$, são estimados os coeficientes do modelo teórico para o semivariograma (o efeito pepita, C0; patamar, $\mathrm{C}_{0}+\mathrm{C}_{1}$; e o alcance, a). Os modelos de semivariogramas considerados foram o esférico, o exponencial, linear com patamar e o gaussiano (McBRATNEY \& WEBSTER, 1986). Para analisar o grau da dependência espacial dos atributos em estudo, utilizou-se a classificação de CAMBARDELLA et al. (1994), em que são considerados de dependência espacial forte os semivariogramas que têm um efeito pepita $<25 \%$ do patamar, moderada quando está entre 25 e $75 \%$, e fraca quando $>75 \%$.

Esses modelos foram ajustados através do programa GS+ (ROBERTSON, 1998). Em caso de divergência entre mais de um modelo para o mesmo semivariograma, considerou-se o maior valor do coeficiente de correlação obtido pelo método de validação cruzada. Os mapas de distribuição espacial foram obtidos por interpolação dos dados (krigagem ordinária) por meio do programa SURFER (GOLDEN SOFTWARE, 1999). Para correlacionar a produtividade da cultura do café com os atributos químicos do solo, utilizou-se o índice de correlação linear (nível de 5\% de significância).

\section{RESULTADOS E DISCUSSÃO}

Os resultados referentes à análise descritiva para os atributos químicos e a produção da cultura do café estão apresentados na (Tabela 1). O teste Kolmogorov-Smirnov indicou normalidade para cálcio, soma de bases e CTC efetiva. Com exceção das variáveis fósforo e produção, os valores da média e mediana, para todas as variáveis, estão próximos, mostrando distribuições simétricas, o que pode ser confirmado pelos valores de assimetria próximos de zero. CORÁ et al. (2004), estudando a variabilidade espacial de atributos químicos e granulométricos do solo para adoção da agricultura de precisão, encontraram normalidade apenas para variável CTC.

Mais importante que a normalidade dos dados é a ocorrência ou não do chamado efeito proporcional, isto é, que a média e a variabilidade dos dados sejam constantes na área de estudo. Esse fato não ocorreu nesta pesquisa, tendo-se em vista que todos os semivariogramas apresentaram patamares bem definidos, e que a distribuição não apresente caudas muito alongadas, o que poderia comprometer as estimativas da krigagem, as quais são baseadas nos valores médios (ISAAKS \& SRIVASTAVA, 1989).

Coeficientes de variação (CV) são adimensionais e permitem a comparação de valores entre diferentes atributos do solo (Tabela 1). Valores elevados de CV podem ser considerados como os primeiros indicadores da existência de heterogeneidade nos dados (FROGBROOK et al., 2002). O pH apresentou o menor coeficiente de variação $(7,13 \%)$, enquanto a produção da cultura cafeeira apresentou o maior coeficiente de variação (78,57\%). BALASTREIRE et al. (2001), em um estudo do mapeamento da produtividade da cultura do café, também encontrou alto coeficiente de variação para a produção. Adotando o critério de classificação para o coeficiente de variação (CV) proposto por WARRICK \& NIELSEN (1980) para medida estatística de dispersão, seus valores revelaramse como: (a) alto (>24\%), para as variáveis potássio, fósforo e produção; (b) médio (12 a 24\%), cálcio, magnésio e soma de bases; (c) baixo $(<12 \%)$, para as variáveis $\mathrm{pH}$ e CTC efetiva (Tabela 1 ).

Todas as variáveis analisadas apresentaram dependência espacial, expressa pelos modelos de semivariogramas (Tabela 2 e Figura 1). As variáveis em estudo ajustaram-se ao modelo esférico, concordando

Tabela 1 - Estatística descritiva para as variáveis $\mathrm{pH}$, cálcio $\left(\mathrm{cmol}_{\mathrm{c}} \mathrm{dm}^{-3}\right)$, magnésio $\left(\mathrm{cmol}_{\mathrm{c}} \mathrm{dm}^{-3}\right)$, potássio $\left(\mathrm{mg} \mathrm{dm}^{-3}\right)$, fósforo $\left(\mathrm{mg} \mathrm{dm}^{-3}\right)$, soma de bases $\left(\mathrm{cmol}_{\mathrm{c}} \mathrm{dm}^{-3}\right)$, capacidade de troca catiônica $\left(\mathrm{cmol}_{\mathrm{c}} \mathrm{dm}^{-3}\right)$ de amostras coletadas na malha na profundidade de 0-0,2 m e produção da cultura do café $\left(\mathrm{L} \mathrm{planta}^{-1}\right)$.

\begin{tabular}{|c|c|c|c|c|c|c|c|c|}
\hline Estatística & $\mathrm{pH}$ & $\mathrm{Ca}$ & Mg & $\mathrm{K}$ & $\mathrm{P}$ & SB & CTC & Produção \\
\hline Média & 6,62 & 3,99 & 1,28 & 186,10 & 4,18 & 5,75 & 7,47 & 4,81 \\
\hline Mediana & 6,70 & 4,01 & 1,30 & 187,12 & 3,76 & 5,90 & 7,50 & 3,75 \\
\hline Variância & 0,22 & 0,87 & 0,07 & 2906 & 9,30 & 1,16 & 0,52 & 18,20 \\
\hline Assimetria & 0,58 & 0,59 & 0,34 & 0,14 & 2,02 & 0,07 & 0,42 & 2,44 \\
\hline Curtose & $-0,55$ & 1,17 & $-0,09$ & $-0,86$ & 5,42 & 0,14 & 0,22 & 7,41 \\
\hline${ }^{1} \mathrm{CV}$ & 7,13 & 23,12 & 21,15 & 29,12 & 72,83 & 18,73 & 9,67 & 78,57 \\
\hline${ }^{2} \mathrm{~d}$ & 0,19 & $0,10^{\mathrm{ns}}$ & 0,12 & 0,12 & 0,20 & $0,09^{\mathrm{ns}}$ & $0,08^{\text {ns }}$ & 0,21 \\
\hline${ }^{3} \mathrm{CR}$ & 0,11 & 0,07 & 0,12 & 0,52 & $-0,09$ & 0,14 & 0,18 & $-0,70$ \\
\hline
\end{tabular}

${ }^{1} \mathrm{CV}=$ coeficiente de variação; ${ }^{2} \mathrm{~d}=$ teste de normalidade, ${ }^{\mathrm{ns}}$ não significativo pelo teste de Kolmogorov-Smirnov; ${ }^{3} \mathrm{CR}=\mathrm{correlação} \mathrm{da}$ produção com o relevo. 
Tabela 2 - Modelos e parâmetros estimados dos semivariogramas experimentais para as variáveis $\mathrm{pH}$, cálcio $\left(\mathrm{cmol}_{\mathrm{c}} \mathrm{dm}{ }^{-3}\right)$, magnésio $\left(\mathrm{cmol}_{\mathrm{c}}\right.$ $\left.\mathrm{dm}^{-3}\right)$, potássio $\left(\mathrm{mg} \mathrm{dm}^{-3}\right)$, fósforo $\left(\mathrm{mg} \mathrm{dm}^{-3}\right)$, soma de bases $\left(\mathrm{cmol}_{\mathrm{c}} \mathrm{dm}^{-3}\right)$, capacidade de troca catiônica $\left(\mathrm{cmol}_{\mathrm{c}} \mathrm{dm}^{-3}\right)$ de amostras coletadas na malha na profundidade de 0-0,2 m e produção da cultura do café (L planta $\left.{ }^{-1}\right)$.

\begin{tabular}{lcccccccc}
\hline Parâmetro & $\mathrm{pH}$ & $\mathrm{Ca}$ & $\mathrm{Mg}$ & $\mathrm{K}$ & $\mathrm{P}$ & $\mathrm{SB}$ & $\mathrm{CTC}$ & Produção \\
\hline Modelo & Esférico & Esférico & Esférico & Esférico & Esférico & Esférico & Esférico & Esférico \\
Efeito pepita $\left(\mathrm{C}_{0}\right)$ & 0,01 & 0,17 & 0,007 & 1074 & 0,79 & 0,27 & 0,16 & 0,79 \\
Patamar $\left(\mathrm{C}_{0}+\mathrm{C}_{1}\right)$ & 0,12 & 0,71 & 0,03 & 3509 & 2,57 & 0,99 & 0,41 & 8,09 \\
Alcance $(\mathrm{a})$ & 72,91 & 67,42 & 65,35 & 347,82 & 327,92 & 65,2 & 98,45 & 60,43 \\
{$\left[\mathrm{C}_{0} /\left(\mathrm{C}_{0}+\mathrm{C}_{1}\right)\right] \mathrm{x} 100$} & 8 & 23 & 23 & 30 & 31 & 27 & 39 & 10 \\
${ }^{1} \mathrm{VC}$ & 0,85 & 0,88 & 0,89 & 0,90 & 0,91 & 0,90 & 0,89 \\
${ }^{2} \mathrm{R}^{2}$ & 67 & 91 & 0,68 & 94 & 92 & 82 & 81 \\
\hline
\end{tabular}

${ }^{1} \mathrm{VC}=$ coeficiente de determinação do teste de validação cruzada; ${ }^{2} \mathrm{R}^{2}=$ coeficiente de determinação.

com os resultados de várias pesquisas que indicam o modelo esférico como o de maior ocorrência para os atributos do solo e de plantas (SOUZA et al., 1997; SOUZA et al., 2004; GREGO \& VIEIRA, 2005). MARQUES JÚNIOR et al. (2000), estudando a variabilidade espacial de atributos químicos e produção do café, ajustaram os dados dos atributos químicos ao modelo esférico, com exceção da variável produção.

O efeito pepita é um parâmetro importante do semivariograma e indica variabilidade não explicada, considerando a distância de amostragem utilizada (McBRATNEY \& WEBSTER, 1986). O efeito pepita foi expresso como percentagem do patamar, com o objetivo de facilitar a comparação do grau de dependência espacial das variáveis em estudo (TRANGMAR et al., 1985). Na análise do grau de dependência espacial das variáveis em estudo, utilizou-se a classificação de CAMBARDELLA et al. (1994). A análise da relação C0/(C0+C1) das variáveis $\mathrm{pH}$, cálcio, magnésio e produção mostrou que as variáveis apresentaram dependência espacial forte e as variáveis potássio, fósforo, soma de bases e CTC efetiva apresentaram dependência espacial moderada (Tabela 2).

Os valores do alcance relativos aos semivariogramas têm uma importância considerável na determinação do limite da dependência espacial, podendo ser também um indicativo do intervalo entre unidades de mapeamento de solos (TRANGAMAR et al., 1985; SOUZA et al., 1997; GREGO \& VIEIRA, 2005). As variáveis apresentaram diferentes alcances de dependência espacial, sendo que o potássio apresentou o maior alcance $(347,82 \mathrm{~m})$ e a produção da cultura do café apresentou o menor alcance $(60,43 \mathrm{~m})$. As variáveis $\mathrm{pH}, \mathrm{Ca}, \mathrm{Mg}$, SB e produção da cultura do café apresentaram alcances na ordem de $70 \mathrm{~m}$, CTC efetiva com alcance na ordem de $98 \mathrm{~m}$ e as variáveis fósforo e potássio com alcance na ordem de 340m. Todas as variáveis em estudo apresentaram valores baixos de alcance, com exceção das variáveis fósforo e potássio, evidenciando a baixa continuidade estrutural do solo sob cafeeiro. Quanto menor o alcance, mais rapidamente é obtida a independência entre amostras, uma vez que o alcance é a distância limite da dependência espacial. A variabilidade extrínseca, relativa às práticas de manejo do solo, contribui para a redução do alcance.

Os mapas das variáveis $\mathrm{pH}$, cálcio, magnésio, potássio, fósforo, soma de bases, CTC efetiva e produção da cultura do café apresentaram valores variando de $6,1-7,3 ; 2,6-5,4 \mathrm{cmol}_{\mathrm{c}} \mathrm{dm}^{-3 ;} 1,1-$ $1,5 \mathrm{cmol}_{\mathrm{c}} \mathrm{dm}^{-3}$; $135-235 \mathrm{mg} \mathrm{dm}^{-3} ; 1,8-4,6 \mathrm{mg} \mathrm{dm}^{-3} ; 3,8-$ $7,0 \mathrm{cmol}_{\mathrm{c}} \mathrm{dm}^{-3,} 6,5-8,1 \mathrm{cmol}_{\mathrm{c}} \mathrm{dm}^{-3}$ e $0,5-10,9 \mathrm{~L}_{\text {planta }}{ }^{-1}$, respectivamente (Figura 2). Observa-se uma grande amplitude nos atributos químicos estudados. Esta grande amplitude revela os problemas que podem ocorrer quando se usa a média dos valores para o manejo da fertilidade. Em alguns locais da área, a aplicação de fertilizante será inferior à dosagem necessária; em outros, a aplicação será condizente com as necessidades e, em outros, poderá haver aplicação excessiva.

Verifica-se que, para a maior parte dos atributos químicos do solo, os coeficientes de correlação (Tabela 1) resultaram em valores baixos $(<0,5)$, concordando com os resultados obtidos por MOLIN et al. (2002). As variáveis cálcio e relevo apresentaram os coeficientes de correlação mais elevados acima de 0,50 . A declividade do terreno revelou uma correlação negativa com a produção, apresentando um coeficiente de correlação de -0,70. Isso indica que, nas áreas menos elevadas do talhão, a produtividade é maior.

Estudos têm demonstrado que o relevo tem sido um parâmetro que define a distribuição de atributos químicos do solo ao longo da paisagem e tem apresentado altas correlações com a produtividade de 


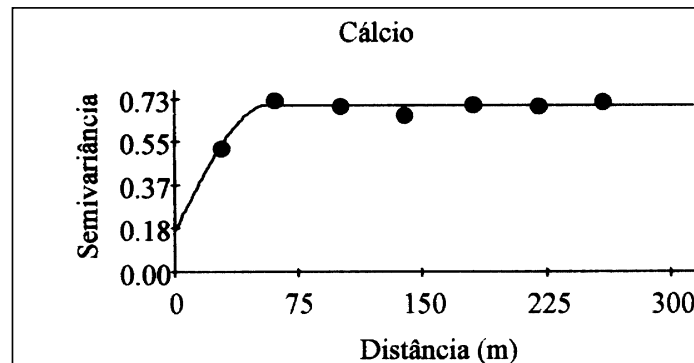

Esf $(0,17-0,71-67,4)$

Potássio

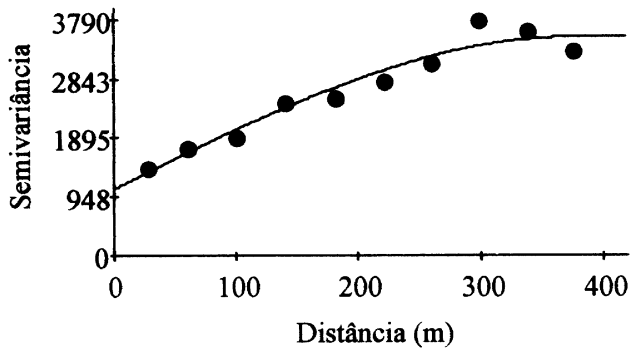

Esf (1074-3509-347,8)

Capacidade de Troca Catiônica

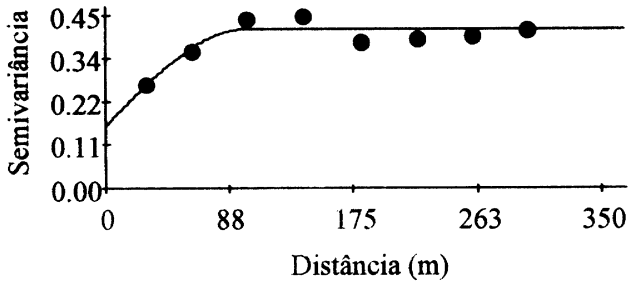

Esf $(0,16-0,41-98,4)$

$\mathrm{pH}$

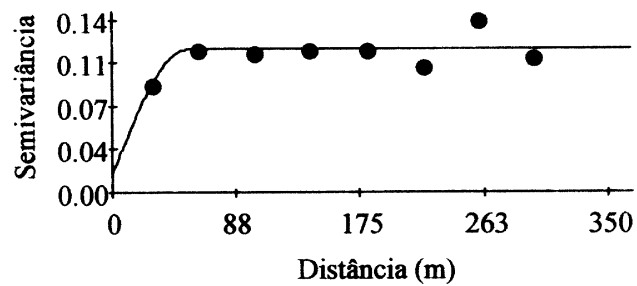

Esf $(0,01-0,12-72,9)$

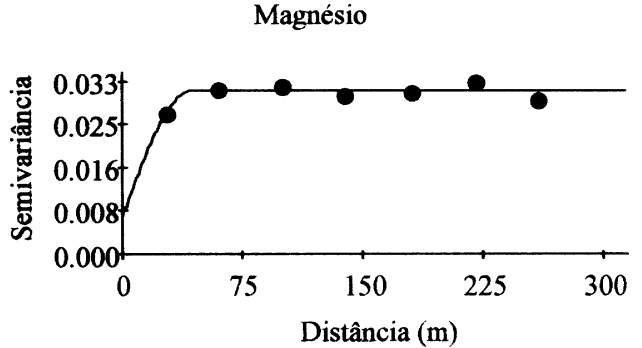

Esf $(0,007-0,03-65,3)$

Fósforo

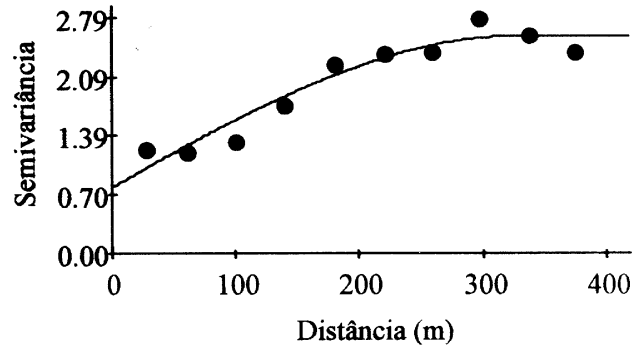

Esf (0,79-2,57-327,9)

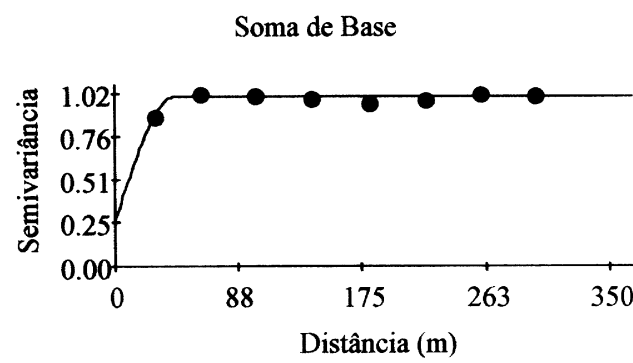

Esf $(0,27-0,99-62,4)$

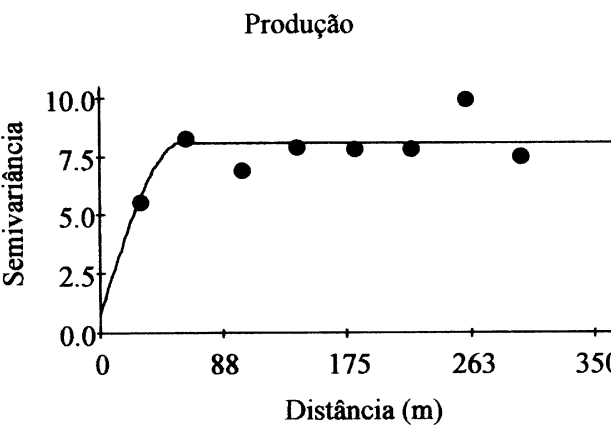

Esf $(0,79-8,09-60,4)$

Figura 1 - Semivariogramas das variáveis químicas: cálcio $\left(\mathrm{cmol}_{\mathrm{c}} \mathrm{dm}^{-3}\right)$, magnésio $\left(\mathrm{cmol}_{\mathrm{c}} \mathrm{dm}^{-3}\right)$, potássio $\left(\mathrm{mg} \mathrm{dm}^{-3}\right)$, fósforo

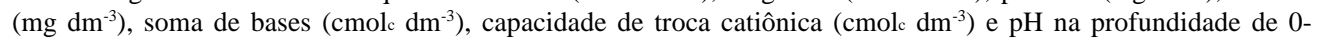
$0,2 \mathrm{~m}$ e produção da cultura do café $\left(\mathrm{L}\right.$ planta $\left.^{-1}\right)$. Esf $\left(\mathrm{C}_{0} ; \mathrm{C}_{0}+\mathrm{C}_{1}\right.$; a) é o modelo esférico ajustado; $\mathrm{C}_{0}=$ efeito pepita; $\mathrm{C}_{0}+\mathrm{C}_{1}=$ patamar; $\mathrm{a}=$ alcance. 


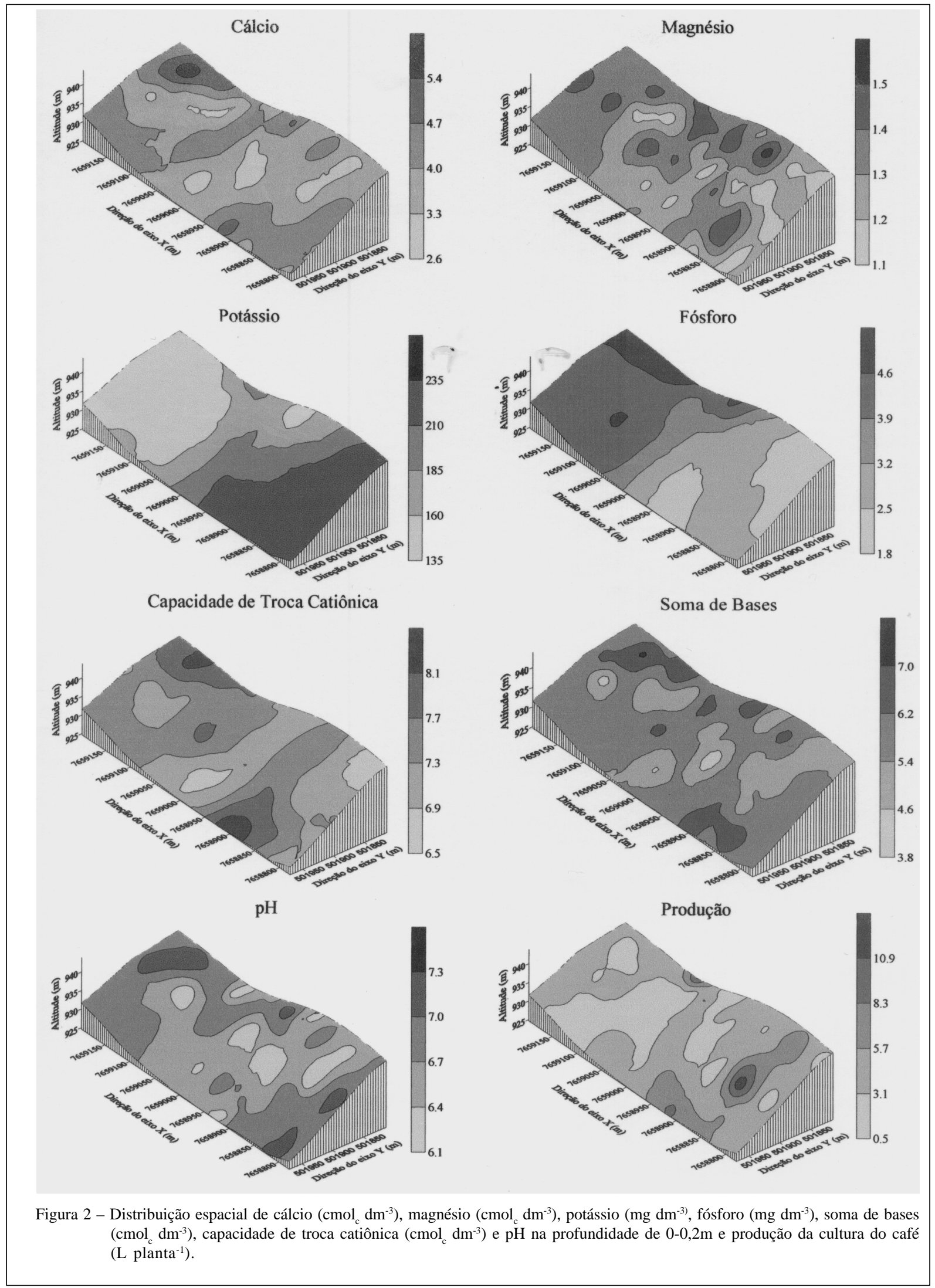

Ciência Rural, v.37, n.2, mar-abr, 2007. 
culturas e atributos do solo (KRAVCHENCO \& BULLOCK, 2000; SOUZA et al., 2003; SOUZA et al., 2004). KUZYAKOVA\& RICHTER (2003), estudando a variabilidade de atributos do solo em um Luvissolo por meio da geoestatística, encontram correlação de atributos químicos do solo com o relevo superior a 0,60 .

\section{CONCLUSÃO}

Todas as variáveis estudadas apresentaram estrutura de dependência espacial, o que permitiu o seu mapeamento, utilizando-se técnicas geoestatísticas. Os atributos químicos apresentaram valores baixos de alcance, com exceção das variáveis fósforo e potássio, evidenciando a baixa continuidade do solo sob cafeeiro. A grande amplitude nos atributos químicos justifica a aplicação diferenciada e localizada de fertilizantes na lavoura cafeeira.

\section{AGRADECIMENTOS}

À FAPEMIG, agência financiadora deste trabalho de pesquisa e da bolsa de recém-doutor do pesquisador Zigomar Menezes de Souza e ao Conselho Nacional de desenvolvimento Científico e Tecnológico (CNPq), pela concessão de bolda de iniciação Científica ai pesquisador Carlos Augusto Pereira de Figueiredo.

\section{REFERÊNCIAS}

BALASTREIRE, L.A. Agricultura de precisão: mapeamento da produtividade de uma cultura de café (compact disc). In CONGRESSO BRASILEIRO DE ENGENHARIA AGRÍCOLA, 30., Foz do Iguaçu, 2001. Anais... Jaboticabal: SBEA, 2001. CD-ROM.

CAMBARDELLA, C.A. et al. Field-scale variability of soil properties in Central Iowa. Soil Science Society of American Journal, Madison, v.58, n.5, p.1501-1511, 1994

CONAB. Companhia nacional de abastecimento. Primeira previsão de safra de café 2005/2006. Brasília, 2006. 15p.

CORÁ, J.E. et al. Variabilidade espacial de atributos do solo para adoção do sistema de agricultura de precisão na cultura de cana-de-açúcar. Revista Brasileira de Ciência do Solo, v.28, n.6, p.1013-1021, 2004.

EMPRESA BRASILEIRA DE PESQUISA AGROPECUÁRIA EMBRAPA. Manual de métodos de análise de solo. 2.ed. Rio de Janeiro: Ministério da Agricultura e do Abastecimento, 1997. 212p.

FROGBROOK, Z.L. et al. Exploring the spatial relations between cereal yield and soil chemical properties and the implications for sampling. Soil Use and Management, Oxon, v.18, n.1, p.1-9, 2002

GREGO, C.R.; VIEIRA, S.R. Variabilidade espacial de propriedades físicas do solo em uma parcela experimental. Revista Brasileira de Ciência do Solo, Viçosa, v.29, n.2, p.169-177, 2005.
ISAAKS, E.H.; SRIVASTAVA, R.M. An introduction to applied geoestatistics. New York: Oxford University, 1989. 561p.

KRAVCHENKO, A.N.; BOLLOCK, D.G. Correlation of corn and soybean yield with topography and soil properties. Agronomy Journal, Madison, v.75, n.1, p.75-83, 2000.

KUZYAKOVA, I; RICHTER, C. Variability of soil parameters in a uniformity trial on a Luvisol evaluated by means of spatial statistics. Journal of Plant Nutrition and Soil Science, Weinheim, v.166, n.3, p.348-356, 2003.

MARQUES JÚNIOR et al. Variabilidade espacial de propriedades químicas e físicas de latossolos em áreas de cerrado sob cultivo de café, em Patrocínio, MG. In: BALASTREIRE, L.A. O estado-da-arte da agricultura de precisão no Brasil, Capítulo III - Mapeamento da Produtividade e de Atributos de Solos e de Plantas. Piracicaba: ESALQ, 2000. p.105-112.

McBRATNEY, A.B.; WEBSTER, R. Choosing functions for semi-variograms of soil properties and fitting them to sampling estimates. Journal Soil Science, Oxford, v.37, n.3, p.617639, 1986

MOLIN, J.P. et al. Mapeamento da produtividade de café e sua correlação com componentes de fertilidade do solo em duas áreas pilotos. In: BALASTREIRE, L.A. Avanços na agricultura de precisão no Brasil no período de 19992001. Piracicaba, ESALQ, 2002. p.58-65.

ROBERTSON, G.P. GS+: geostatistics for the environmental sciences (version 5.1 for windows). Plainwell: Gamma Design Soffware, 1998. 152p.

ROCHA, J.V.; LAMPARELLI, R.A.C. Geoprocessamento. In: SILVA, F.M. Mecanização e agricultura de precisão. Poços de Caldas: UFV, 1998. Cap.1, p.1-30.

SCHLOTZHAVER, S.D.; LITTELL, R.C. SAS: system for elementary statistical analysis. 2.ed. Cary, 1997. 905p.

SOUZA, L.S. et al. Variabilidade de propriedades físicas e químicas do solo em um pomar cítrico. Revista Brasileira de Ciência do Solo, v.21, p.367-372, 1997.

SOUZA, C.K. et al. Influência do relevo na variação anisotrópica dos atributos químicos e granulométricos de uma latossolo em Jaboticabal-SP. Engenharia Agrícola, Jaboticabal, v.23, n.3, p.486-495, 2003.

SOUZA, Z.M. et al. Variabilidade espacial do $\mathrm{pH}$, Ca, Mg e V\% do solo em diferentes formas do relevo sob cultivo de cana-deaçúcar. Ciência Rural, Santa Maria, v.34, n.6, p.1763-1771, 2004.

SURFER for windows. Realese 7.0. Contouring and 3D surface mapping for scientist's engineers. User's guide. New York: Golden software, 1999. 619p.

TRANGMAR, B.B. et al. Applications of geostatistics to spatial studies of soil properties. Advances in Agronomy, San Diego, v.38, n.1, p.45-94, 1985.

VIEIRA, S.R. et al. Geoestatiscal theory and application to variability of some agronomical properties. Hilgardia, Oakland, v.51, n.1, p.1-75, 1983.

WARRICK, A.W.; NIELSEN, D.R. Spatial variability of soil physical properties in the field. In: HILLEL, D. (Ed). Applications of soil physics. New York: Academic, 1980. Cap.2, p.319-344. 